\title{
Berry phase and anomalous transport of the composite fermions at the half-filled Landau level
}

\begin{abstract}
W. Pan ${ }^{1 \star}$, W. Kang ${ }^{2 \star}$, K. W. Baldwin ${ }^{3}$, K. W. West ${ }^{3}$, L.
The fractional quantum Hall effect (FQHE) ${ }^{1,2}$ in twodimensional electron systems is an exotic, superfluid-like matter with an emergent topological order. From the consideration of the Aharonov-Bohm interaction between electrons and magnetic field, the ground state of a half-filled lowest Landau level is mathematically transformed to a Fermi sea of composite objects of electrons bound to two flux quanta, termed composite fermions $\left(\right.$ CFs $^{3-5}$. A strong support for the CF theories comes from experimental confirmation of the predicted Fermi surface at $v=1 / 2$ (where $v$ is the Landau level filling factor) from the detection of the Fermi wavevector in semi-classical geometrical resonance experiments ${ }^{2,6-9}$. Recent developments in the theory of $\mathrm{CFs}^{10-21}$ have led to the prediction of a $\pi$ Berry phase for the CF circling around the Fermi surface at half-filling ${ }^{10,14,17-20}$. In this paper we provide experimental evidence for the detection of the Berry phase of CFs in the fractional quantum Hall effect. Our measurements of the Shubnikov-de Haas oscillations of CFs as a function carrier density at a fixed magnetic field provide strong support for the existence of a $\pi$ Berry phase at $v=1 / 2$. We also discover that the conductivity of composite fermions at $v=1 / 2$ displays an anomalous linear density dependence, whose origin remains mysterious yet tantalizing.
\end{abstract}

Under the framework of the CF theory ${ }^{3-5}$, the effective magnetic field, $B_{\text {eff }}$, that the CFs experience in the lowest Landau level is reduced due to flux attachment so that $B_{\text {eff }}=B-B_{v=1 / 2}$. At half-filling of the Landau level $(v=1 / 2), B_{\text {eff }}$ becomes zero and CFs form a Fermi sea state. This startling prediction of the CF theory has been verified experimentally ${ }^{2,6-9}$. Away from $v=1 / 2$, the $v=n /(2 n+1)$, where $n= \pm 1, \pm 2, \pm 3 \ldots, \mathrm{FQHE}$ states (also called the Jain sequence of FQHE states) can be mapped into the $v^{*}=n$ integer quantum Hall effect (IQHE) states of CFs (Supplementary Fig. 1). The experimentally observed sequence of FQHE states can be viewed as the density of states oscillations of the pseudoLandau levels of CFs under increasing $B_{\text {eff }}$ (refs 3-5). The CF theory has been shown to be extremely proficient in constructing the wavefunctions of the various $\mathrm{FQHE}$ states and in providing explanations of experiments on the $\mathrm{FQHE}^{2,4}$.

There have been new, interesting developments in the study of $\mathrm{CF}$ in recent years. The interest in $\mathrm{CF}$ was reinvigorated in part from a realization of the significance of particle-hole symmetry $^{10,15-18,20-23}$. In particular, a recent experiment on the commensurability oscillations of CFs observed that the oscillation minima above and below half-filling are not symmetric with changing magnetic field ${ }^{23}$. This result hinted that the nature of the composite fermions may change from 'particle-like' below to 'hole-like' above half-filling. Whereas particle-hole symmetry at half-filling had been accepted without question, its experimental consequence on the CFs at half-filling remained largely unexplored.

Many theoretical studies of the particle-hole symmetry in the FQHE predict a Berry phase of the composite Fermi liquid at $\nu=1 / 2$ (refs 10,17-20). In the Dirac theory of CFs at finite density $^{10-14}$, the CFs at half-filling are treated as Dirac fermions with a $\pi$ Berry phase from its motion about the Fermi surface. An alternate approach, which considers CFs as charge-neutral particle carrying vorticity $^{17,18}$, associates a Berry curvature about the CF Fermi surface at half-filling. A microscopic approach to CFs based on geometrical considerations also predicts a Berry phase for adiabatic transport of CFs around the Fermi surface ${ }^{19,24}$. The connection between the Dirac theory ${ }^{10-14}$ and the Halperin, Lee, Read (HLR) theory ${ }^{3}$ of CFs was explored recently $y^{20}$. These theories provide various perspectives into the physics of CFs at the half-filled Landau level. The prediction of a Berry phase for CFs in the FQHE is a significant new development, as its possibility has been overlooked in the past.

In this paper we study CFs at the half-filled Landau level by studying their density-dependent magnetotransport. To look for signatures of the predicted Berry phase, we have measured the density-dependent Shubnikov-de Haas oscillations of magnetoresistivity, $\rho_{x x}$, around $v=1 / 2$ at various magnetic fields in two heterojunction insulated-gate field-effect transistors (HIGFETs) ${ }^{25}$, in which the electron density $\left(n_{\mathrm{e}}\right)$ can be tuned over a large range. (See details of the characterizations in Supplementary Fig. 2.) Study of SdH oscillations in the FQHE has been a very useful probe of CFs in determining their effective mass in the lowest Landau level ${ }^{26,27}$. In the case of graphene, studies of $\mathrm{SdH}$ oscillations have been successfully utilized to detect the Berry phase of Dirac fermions about the Dirac cone ${ }^{28,29}$. Our Berry phase results can be understood within the framework of the recently proposed theoretical studies of CFs at the half-filled Landau level ${ }^{10-21}$.

Figure 1a shows the $\mathrm{SdH}$ oscillations from specimen HIGFET-A as a function of $n_{\mathrm{e}}$ under a fixed magnetic field with $B=10 \mathrm{~T}$. A series of well-defined FQHE states is observable as a function of density as $\mathrm{SdH}$ oscillation minima around $v=1 / 2$. Under a fixed magnetic field $B$, the density-dependent $\mathrm{SdH}$ oscillation of the magnetoresistivity, $\Delta \rho_{x x}$, can be modelled as

$$
\Delta \rho_{x x}=R\left(n_{\mathrm{e}}, T\right) \cos \left[2 \pi\left(\frac{n_{\mathrm{B}}}{n_{1 / 2}-n_{\mathrm{e}}}+\beta\right)\right]
$$

where $R\left(n_{\mathrm{e}}, T\right)$ is the amplitude of the $\mathrm{SdH}$ oscillations as a function of density and temperature, $n_{1 / 2}$ is the density at $v=1 / 2$ for the given $B, n_{\mathrm{B}}$ the frequency of the density-dependent $\mathrm{SdH}$ oscillations, and $\beta$ the associated Berry phase of the CF motion about the Fermi surface. In our analysis we first locate the densities corresponding

\footnotetext{
${ }^{1}$ Sandia National Laboratories, Albuquerque, New Mexico 87185, USA. ²Department of Physics, University of Chicago, Chicago, Illinois 60637, USA.

${ }^{3}$ Department of Electrical Engineering, Princeton University, Princeton, New Jersey 08544, USA. *e-mail: wpan@sandia.gov; wkang@uchicago.edu
} 
to the FQHE states at the magnetoresistivity minima of the $\mathrm{SdH}$ oscillations. (The $\rho_{x x}$ maxima can be neglected since only the FQHE states at the minima are relevant for CFs.) The value of the index $n$ for the respective $\mathrm{SdH}$ minima can be independently determined from the one-to-one correspondence for the Jain states between the IQHE around $B=0$ and the FQHE around $v=1 / 2$. The procedure is illustrated in Supplementary Fig. 1. As shown in Supplementary Fig. 1, after the $R_{x x}$ curve is horizontally shifted so that the magnetic field position at $v=1 / 2$ overlaps with $B=0$, we observe that the $v=1 / 3$ and 1 states, the $2 / 5$ and 2 states, the $3 / 7$ and 3 states, and so on respectively occur at the same positions. Based on this observation, one can assign an effective integer filling factor to a FQHE state, that is, filling factor 1 to the $1 / 3$ state, 2 to $2 / 5,3$ to $3 / 7$, and -2 to $2 / 3,-3$ to $3 / 5,-4$ to $4 / 7$, and so on. The effective magnetic field $B_{n}^{*}=B-B_{1 / 2}\left(n_{\mathrm{e}}\right)$, where $B_{1 / 2}\left(n_{\mathrm{e}}\right)=2 n_{\mathrm{e}} h / e$ is the magnetic field at half-filling for an electron density of $n_{\mathrm{e}}$. The inset of Fig. $1 \mathrm{~b}$ illustrates how $B_{n}^{*}$ is determined in such a density sweep. The value of $B_{n}^{*}$ is shown as the top axis in Fig. 1a. In Fig. 1b, we plot the $\mathrm{SdH}$ oscillation index $n$ versus $1 / B_{n}^{*}$. An intercept of $\beta=-1 / 2$ at $1 / B_{n}^{*}=0$ is clearly seen in Fig. 1 b. This result shows a Berry phase of $\pi$ for the CFs at $\nu=1 / 2$.

We point out that a similar measurement has been carried out in graphene to characterize its Berry phase ${ }^{28,29}$. The chiral nature of massless Dirac fermions in graphene produces a Berry phase of $\pi$ about its Dirac point, resulting in SdH oscillations that are phase shifted by $\pi$ relative to two-dimensional (2D) systems with conventional Fermi surfaces. Experiments have shown that $\beta$ determined from the intercept is $-1 / 2$ in graphene. Our measurement likewise illustrates that the density-dependent $\mathrm{SdH}$ oscillations of the CFs demonstrate that $\beta=-1 / 2$, which corresponds to a $\pi$ Berry phase about the Fermi surface at $v=1 / 2$. We note here that in the $\mathrm{SdH}$ measurements at fixed electron density, the intercept at $1 / B_{n}=0$ is zero, yielding a zero for the Berry phase. Here $B_{n}$ is defined as $B_{n}=B_{v}-B_{1 / 2}$ for the effective magnetic field that CFs see in standard $\mathrm{SdH}$ oscillations where the external $B$ field is varied. We have shown this in one of our high-mobility quantum well samples in Supplementary Fig. 4.

We also point out that in the proposals of Dirac composite fermions at finite density ${ }^{10-14}$ and CFs as neutral particles carrying vorticity $^{17,18}$, the CF density, $n_{v}$, is proportional to the external magnetic field, $n_{v}=e B / 2 h$. Only at $v=1 / 2$ does $n_{v}$ equal the underlying electron density $\left(n_{\mathrm{e}}\right)$. In the HLR theory of CFs, the density of composite fermions equals the electron density. It was pointed out by Wang and Senthil that in conventional SdH measurements at fixed density, both the CF density and its effective magnetic field change with varying external magnetic field ${ }^{18}$. Thus it is not possible to obtain a coherent Berry phase, and $\beta$ becomes zero in $\mathrm{SdH}$ measurements at fixed density.

Figure 2 summarizes the measurement and analysis of densitydependent $\mathrm{SdH}$ oscillations at different values of fixed magnetic field. Figure $2 \mathrm{a}-\mathrm{c}$ shows $\mathrm{SdH}$ oscillations about $v=1 / 2$ for three different magnetic fields. Figure $2 \mathrm{~d}$ shows the resulting $\mathrm{SdH}$ fan diagram of $n$ versus $1 / B_{n}^{*}$ for the density sweeps in Fig. $2 \mathrm{a}-\mathrm{c}$, where all the data converge at a vertical intercept of $\beta=-1 / 2$. A summary of slope intercepts $\beta$ determined from the various density sweeps is shown in Fig. 2e. The slope intercepts from the density sweeps with different magnetic fields all universally possess a $\beta=-1 / 2$, confirming the predicted Berry phase of $\pi$ for the CFs at $\nu=1 / 2$.

We now turn our attention to the $\nu=3 / 2$ state, the particle-hole conjugate state of the $1 / 2$ state. Around $v=3 / 2$, the FQHE occurs at $v=2-n /(2 n+1)$. We measure the $\mathrm{SdH}$ oscillations as a function of electron density at a fixed magnetic field and apply the formula $B_{n}^{*}=3 \times\left(B-2 h / 3 e \times n_{\mathrm{e}}\right)$ for the $v=3 / 2$ CFs. With this definition of effective magnetic field, we arrive at $n=-B / 2 \times 1 / B_{n}^{*}-1 / 2$, the same as the $v=1 / 2$ CFs. We note here that the minus sign in front of $B / 2$ reflects the hole nature of the CFs at $v=3 / 2$. a
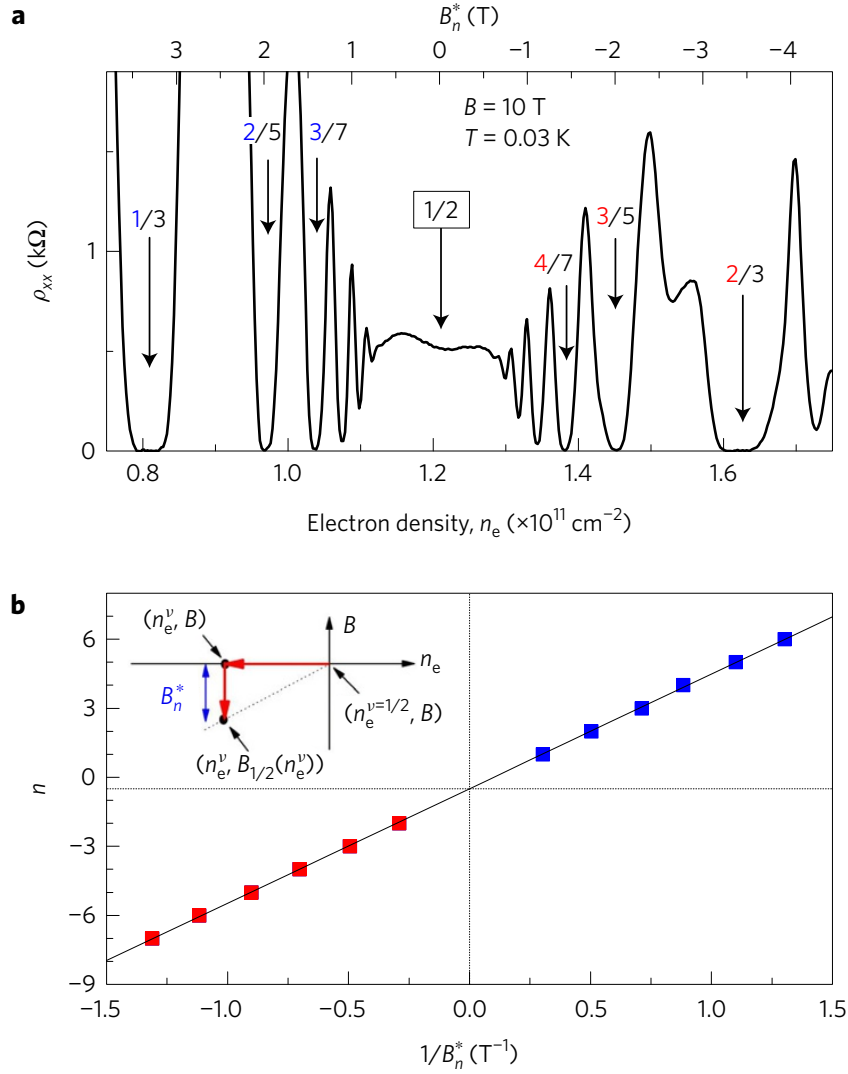

Figure 1 | Composite fermion Shubnikov-de Haas oscillations and Landau fan diagram in a heterojunction insulated-gate field-effect transistor (HIGFET). a, $\rho_{x x}$ as a function of electron density $\left(n_{\mathrm{e}}\right.$, in units of $10^{11} \mathrm{~cm}^{-2}$ ). The arrows mark several representative FQHE states. Their corresponding CF pseudo-Landau level filling factor $n$ is indicated with the coloured numerator, blue for positive and red for negative integers. The index $n$ is determined independently from the one-to-one correspondence between the FQHE around $v=1 / 2$ and the IQHE around $B=0$, as shown in Supplementary Fig. 1. For every state with index $n$, the corresponding filling factor under the composite fermion theory is given by $v=n /(2 n+1)$. The upper $x$ axis shows the value of $B_{n}^{*}=B-2 n_{\mathrm{e}} h / e$. We note that the particle-hole conjugate states-for example, the $1 / 3(n=1)$ and $2 / 3$ $(n=-2)$ states-are located equal densities away from $v=1 / 2$. b, CF pseudo-Landau level filling factor $n$ versus $1 / B_{n}^{*}$. Positive values of $n$ are shown in blue and negative $n$ values are shown in red. The inset of $\mathbf{b}$ illustrates how $B_{n}^{*}$ is determined in a density sweep.

Figure 3a shows the $\mathrm{SdH}$ oscillations around $v=3 / 2$ as a function of electron density at a fixed magnetic field of 5.0 T. The arrows mark a few representative FQHE states. In Fig. 3 b, we plot $n$ versus $1 / B_{n}^{*}$. An intercept of $-1 / 2$ at $1 / B_{n}^{*}=0$ is clearly seen, now establishing direct detection of a $\pi$-Berry phase for the CFs at $v=3 / 2$. A summary of the slope intercepts determined from the various density sweeps with different magnetic fields is shown in Supplementary Fig. 5. They all universally possess the value of $-1 / 2$.

For CFs around $v=3 / 2$, in standard, $B$-dependent $\mathrm{SdH}$ oscillations, the effective magnetic field of CFs around $v=3 / 2$ is given by $B_{n}=3 \times\left(B_{v}-B_{3 / 2}\right)=-n_{\mathrm{e}} h / e \times 1 /(3 n+2)$, or $n=-n_{\mathrm{e}} h / 3 e \times 1 / B_{n}-2 / 3$. The intercept of $-2 / 3$ was indeed observed in the standard $\mathrm{SdH}$ oscillations, as shown in Supplementary Fig. 6.

In our density-dependent $\mathrm{SdH}$ measurement, the density of CFs depends on the magnetic field at $v=1 / 2$. The CF density, $n_{v}$, is proportional to the external magnetic field and only at $v=1 / 2$ equals the underlying electron density $n_{\mathrm{e}}$. Our experimental detection 
a

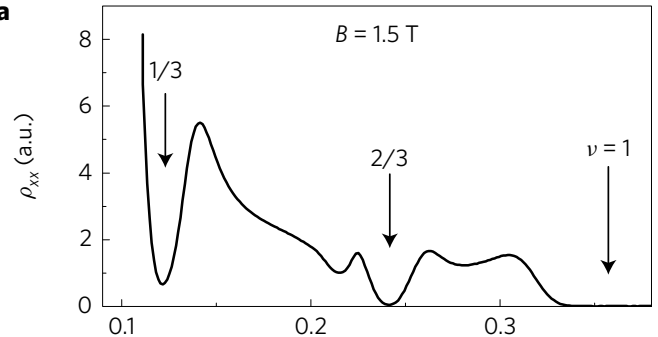

b

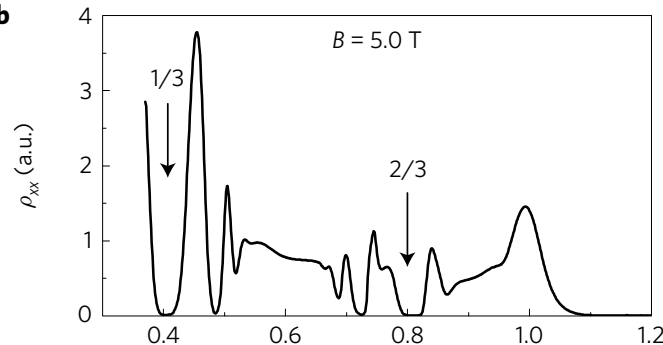

c

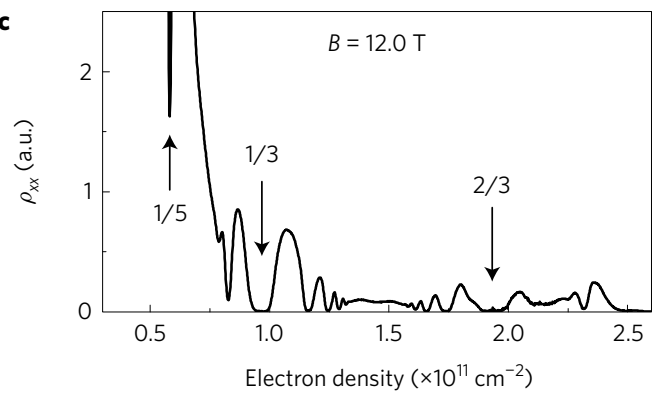

d

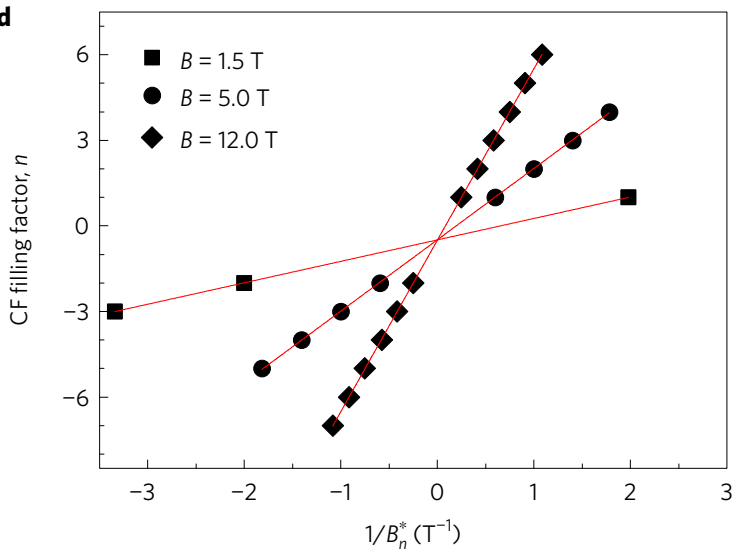

e

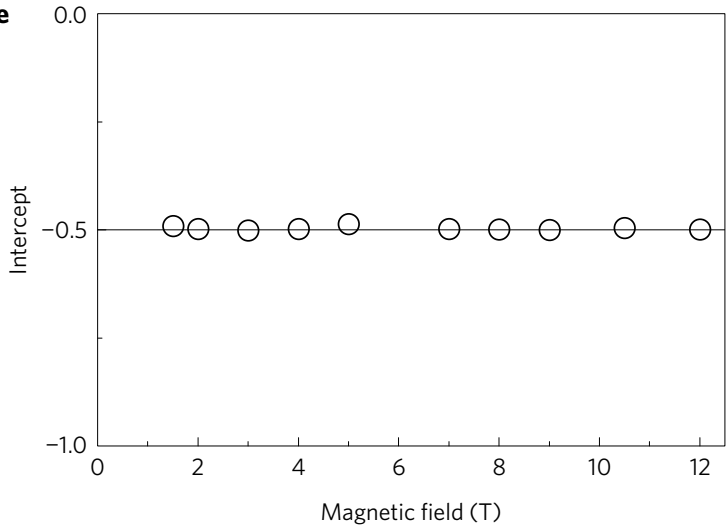

Figure 2 Shubnikov-de Haas oscillations around $v=1 / 2$ as a function of electron density $\left(n_{\mathrm{e}}\right.$, in units of $10^{11} \mathrm{~cm}^{-2}$ ) in HIGFET-B at a few selected magnetic fields. $\mathbf{a}, B=1.5 \mathrm{~T}$. b, $5.0 \mathrm{~T}$. c, $12.0 \mathrm{~T}$. The arrows mark the Landau level filling factors at $v=1 / 3,2 / 3$, and 1, 1/5, respectively. d, CF pseudo-Landau level filling factor $n$ versus $1 / B_{n}^{*}$, where $B_{n}^{*}=B-2 n_{\mathrm{e}} h / e$. e, Plot of the intercept at $1 / B_{n}^{*}=0$ in $\mathbf{d}$ versus the external magnetic field.

of the Berry phase in the density-dependent $\mathrm{SdH}$ measurement appears to support the proposal that the CF density is proportional to $B_{1 / 2}$, the magnetic field at $v=1 / 2$, and not the electron density. However, our observation of the Berry phase cannot be viewed as evidence of the Dirac fermions ${ }^{10-14}$ or the neutral vortex theories of $\mathrm{CF}^{17,18}$. The Berry phase at $v=1 / 2$ appears to be an emergent, universal feature of the underlying CF liquid ${ }^{10-24}$ as electrons seek to minimize their interaction energy in the lowest Landau level.

In contrast to similar experiments in graphene, a Dirac point in the lowest Landau level cannot be directly observed in GaAs heterostructures or similar systems. In the context of the Dirac fermion theory of $\mathrm{CFs}^{10-14}$, an effective Dirac point may be discussed as an extrapolation from higher-lying pseudo-CF Landau levels. Direct detection of such a Dirac point is beyond the scope of the present experiment. It follows that our observation of the $\pi$ Berry phase of CFs establishes an important, previously unrealized correlation of CFs.

In Fig. 4 we present the first ever result for the density dependence of CF conductivity, which in principle provides an important insight into the transport of CFs at $v=1 / 2$. A plot of $1 / \rho_{x x}$ at $v=1 / 2$, obtained in the two HIGFETs, is shown as a function of electron density $n_{\mathrm{e}}$. Since the conductivity of CFs, $\sigma_{\mathrm{CF}} \cong 1 / \rho_{x x} \times\left(1+\left(\rho_{x x} / \rho_{x y}\right)^{2}\right)$ (ref. 17) at $v=1 / 2$, and with $\left(\rho_{x x} / \rho_{x y}\right)^{2}<0.1 \%, \sigma_{\mathrm{CF}} \cong 1 / \rho_{x x}$. Both HIGFETs show that the conductivity of CFs shows a linear dependence over a large density range from $\sim 2 \times 10^{10}$ to $1.4 \times 10^{11} \mathrm{~cm}^{-2}$. A linear dependence of conductivity was also observed for the CFs at $v=3 / 2$, as shown in Supplementary Fig. 7. Differing from the results at $v=1 / 2$ and $3 / 2$, the conductivity of electrons at $B=0$ follows a $n_{\mathrm{e}}^{2}$ density dependence (details shown in Supplementary Fig. 8).
At this point we do not understand the origin of this striking linear density dependence of conductivity at $v=1 / 2$. While a $2 \mathrm{D}$ electron systems in a heterostructure with an appropriate form of disorder may have a conductivity $\sigma_{x x} \sim n^{\alpha}, \alpha \approx 1$ at $\nu=1 / 2$ (ref. 30), it is unclear how well this theory applies to our HIGFET device, which has no doping and instead relies on electrostatic gating.

The observed linear density dependence of conductivity at $\nu=1 / 2$ may be viewed in terms of a constant CF mobility, $\mu^{*}$, with $\sigma_{\mathrm{CF}}=n_{\nu} e \mu^{*}$. Consequently, the scattering time $\tau *=\mu^{*} m^{*} / e \propto n_{v}^{1 / 2}$, since the effective mass of CFs, $m^{*} \propto n_{v}^{1 / 2}$ (ref. 31). This apparent $\tau^{*} \propto n_{v}^{1 / 2}$ behaviour differs from the HLR theory ${ }^{3}$, where the CF scattering time $\tau^{*} \propto m^{*} k_{\mathrm{F}} \propto n_{v}$, since the Fermi wavevector for CFs, $k_{\mathrm{F}} \propto n_{\nu}^{1 / 2}$. Moreover, from the Dingle analysis of the $\mathrm{SdH}$ oscillations around $v=1 / 2$ (Supplementary Fig. 9), it was observed that CF Landau level broadening (or momentum scattering time) shows a weak density dependence. More studies will be needed to understand the origin of this weak density-dependent behaviour and whether it is responsible for the linear density dependence of CF conductivity.

In the context of the Dirac theory of $\mathrm{CFs}^{10-14}$ or the neutral vorticity theory ${ }^{18,19}$, the density of CF at $\nu=1 / 2$ is $n_{v}=e B_{1 / 2} / 2 h$. Then the conductivity at the half-filling, $\sigma_{x x} \sim n_{v} \sim B_{1 / 2}$, provided that $\mu^{*}$ is constant. Interestingly, it was shown experimentally in ultra-clean specimens that longitudinal resistivities at even denominator fillings are linear with magnetic field ${ }^{32,33}$. The observed behaviour is consistent with our results, since $\rho_{x x} \sim \sigma_{x x} \sim B_{1 / 2}$. Our finding therefore hints at the possibility of some novel exotic entanglement of the CFs at half-filling.

It is well known that the conductivity of 2D Dirac fermions-for example, in graphene-displays a linear density dependence, due to 

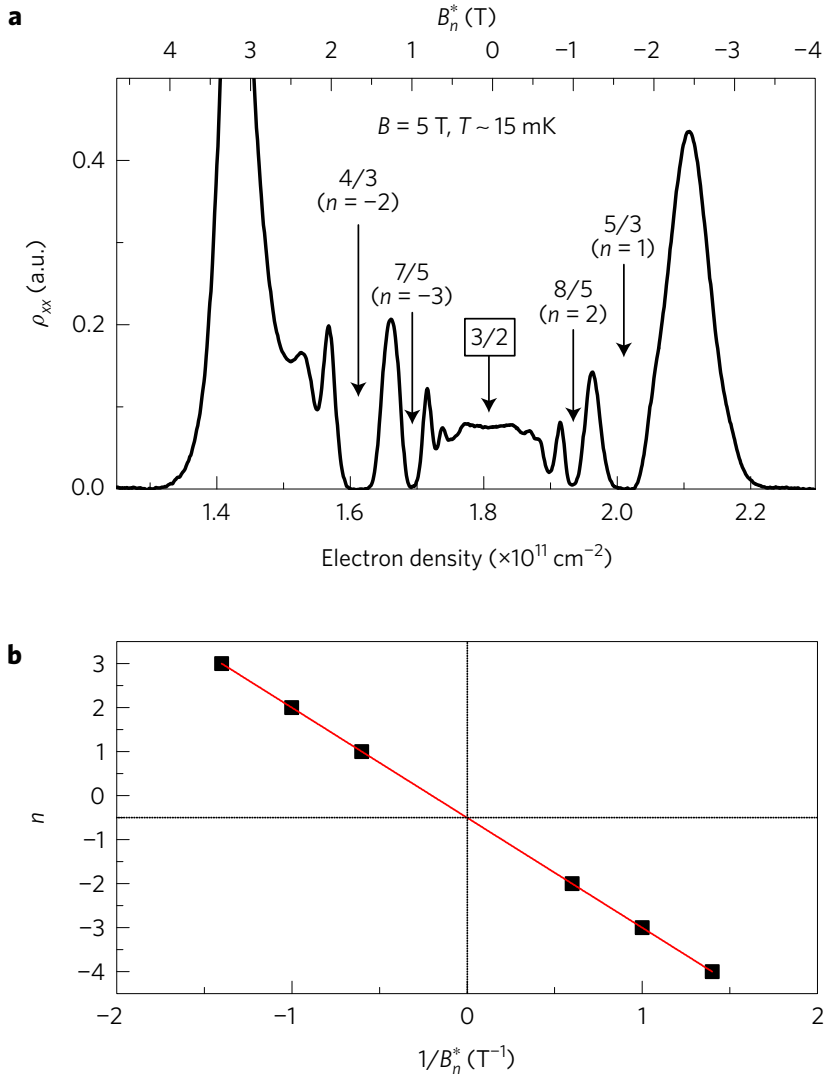

Figure 3 | Composite fermion Shubnikov-de Haas oscillations around $v=3 / 2$ and Landau fan diagram. $\mathbf{a}, \rho_{x x}$ as a function of electron density $\left(n_{e}\right.$, in units of $\left.10^{11} \mathrm{~cm}^{-2}\right) . \mathbf{b}, C F$ pseudo-Landau level filling factor $n$ versus $1 / B_{n}^{*}$. The intercept at $1 / B_{n}^{*}=0$ is $-1 / 2$.

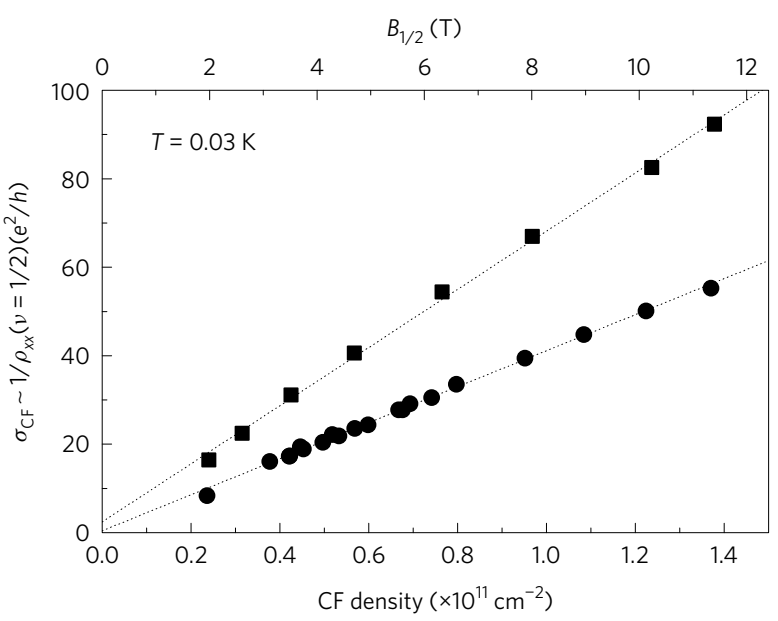

Figure 4 | Conductivity of composite fermions at $v=1 / 2$ in two HIGFETs. The two HIGFETs have different growth structures and, thus, peak mobilities. In the density range between $2 \times 10^{10}$ and $1.4 \times 10^{11} \mathrm{~cm}^{-2}$, the conductivity displays a linear density dependence in both samples.

its linear dispersion ${ }^{28,29}$. Since there is no experimental evidence yet available for the existence of Dirac fermions at $v=1 / 2$, it is unclear if there is any connection between the linear density dependence of graphene and the CFs at $v=1 / 2$ at this time. At minimum, a further clarification of the scattering time will be necessary to better understand this puzzling behaviour of the CFs at the halffilled Landau level.

\section{Methods}

Methods, including statements of data availability and any associated accession codes and references, are available in the online version of this paper.

Received 19 February 2017; accepted 12 July 2017; published online 21 August 2017

\section{References}

1. Tsui, D. C., Stormer, H. L. \& Gossard, A. C. Two-dimensional magnetotransport in the extreme quantum limit. Phys. Rev. Lett. 48, 1559-1562 (1982).

2. Perspectives in Quantum Hall Effects (eds Das Sarma, S. \& Pinczuk, A.) (Wiley Interscience, 1997).

3. Halperin, B. I., Lee, P. A. \& Read, N. Theory of the half-filled Landau level. Phys. Rev. B 47, 7312-7343 (1993).

4. Jain, J. K. Composite-fermion approach for the fractional quantum Hall effect. Phys. Rev. Lett. 63, 199-202 (1989).

5. Lopez, A. \& Fradkin, E. Fractional quantum Hall effect and Chern-Simons gauge theories. Phys. Rev. B 44, 5246-5262 (1991).

6. Willett, R. L., Ruel, R. R., West, K. W. \& Pfeiffer, L. N. Experimental demonstration of a Fermi surface at one-half filling of the lowest Landau level. Phys. Rev. Lett. 65, 3846-3849 (1993).

7. Kang, W., Stormer, H. L., Pfeiffer, L. N., Baldwin, K. W. \& West, K. W. How real are composite fermions? Phys. Rev. Lett. 71, 3850-3853 (1993).

8. Goldman, V. J., Su, B. \& Jain, J. K. Detection of composite fermions by magnetic focusing. Phys. Rev. Lett. 72, 2065-2068 (1994).

9. Smet, J. H. et al. Magnetic focusing of composite fermions through arrays of cavities. Phys. Rev. Lett. 77, 2272-2275 (1996).

10. Son, D. T. Is the composite fermion a Dirac particle? Phys. Rev. X 5, 031027 (2015).

11. Mross, D. F., Essin, A. \& Alicea, J. Composite Dirac liquids: parent states for symmetric surface topological order. Phys. Rev. X 5, 011011 (2015).

12. Metlitski, M. A. \& Vishwanath, A. Particle-vortex duality of 2D Dirac fermion from electric-magnetic duality of 3D topological insulators. Phys. Rev. B 93, 245151 (2016).

13. Geraedts, S. D. et al. The half-filled Landau level: the case for Dirac composite fermions. Science 352, 197-201 (2016).

14. Potter, A. C., Serbyn, M. \& Vishwanath, A. Thermoelectric transport signatures of Dirac composite fermions in the half-filled Landau level. Phys. Rev. X 6, 031026 (2016).

15. Barkeshli, M., Mulligan, M. \& Fisher, M. P. A. Particle-hole symmetry and the composite Fermi liquid. Phys. Rev. B 92, 165125 (2015).

16. Balram, A. C., Toke, C. \& Jain, J. K. Luttinger theorem for the strongly correlated Fermi liquid of composite fermions. Phys. Rev. Lett. 115, 186805 (2015).

17. Wang, C. \& Senthil, T. Half-filled Landau level, topological insulator surfaces, and three dimensional quantum spin liquids. Phys. Rev. B 93, 085110 (2016).

18. Wang, C. \& Senthil, T. Composite Fermi liquids in the lowest Landau level. Phys. Rev. B 94, 245107 (2016).

19. Haldane, F. D. M. A model wavefunction for the Composite Fermi Liquid, its Geometry and Entanglement. Presentation at the 2016 American Physical Society March Meeting; https://absuploads.aps.org/ presentation.cfm?pid $=11640$

20. Wang, C., Cooper, N. R., Halperin, B. I. \& Stern, A. Particle-hole symmetry in the Fermion-Chern-Simons and Dirac descriptions of a half-filled Landau level. Preprint at http://arxiv.org/abs/1701.00007 (2017).

21. Murthy, G. \& Shankar, R. The $v=1 / 2$ Landau level: half-full or half-empty? Phys. Rev. B 93, 085405 (2016).

22. Kivelson, S. A., Lee, D.-H., Krotov, Y. \& Gan, J. Composite-fermion Hall conductance at $v=1 / 2$. Phys. Rev. B 55, 15552-15561 (1997).

23. Kamburov, D. et al. What determines the Fermi wave vector of composite fermions? Phys. Rev. Lett. 113, 196801 (2014).

24. Haldane, F. D. M. Berry curvature on the Fermi surface: anomalous Hall effect as a topological Fermi-liquid property. Phys. Rev. Lett. 93, 206602 (2004).

25. Pan, W., Baldwin, K. W., West, K. W., Pfeiffer, L. N. \& Tsui, D. C. Quantitative examination of the collapse of spin splitting in the quantum Hall regime. Phys. Rev. B 84, 161307(R) (2011).

26. Leadley, D. R., Nicholas, R. J., Foxon, C. T. \& Harris, J. J. Measurements of the effective mass and scattering times of composite fermions from magnetotransport analysis. Phys. Rev. Lett. 72, 1906-1909 (1994).

27. Du, R. R. et al. Drastic enhancement of composite fermion mass near Landau level filling $v=1 / 2$. Phys. Rev. Lett. 73, 3274-3277 (1994).

28. Novoselov, K. S. et al. Two-dimensional gas of massless Dirac fermions in grapheme. Nature 438, 197-200 (2005). 
29. Zhang, Y., Tan, Y.-W., Stormer, H. L. \& Kim, P. Experimental observation of the quantum Hall effect and Berry's phase in grapheme. Nature 438, 201-203 (2005).

30. Mirlin, A. D., Polyakov, D. G. \& Wölfle, P. Composite fermions in a long-range random magnetic field: quantum Hall effect versus Shubnikov-de Haas oscillations. Phys. Rev. Lett. 80, 2429-2432 (1998).

31. Pan, W. et al. Effective mass of the four-flux composite fermion at $v=1 / 4$. Phys. Rev. B 61, R5101(R) (2000).

32. Stormer, H. L., Baldwin, K. W., Pfeiffer, L. N. \& West, K. W. Strikingly linear magnetic field dependence of the magnetoresistivity in high quality two-dimensional electron systems. Solid State Commun. 84, 95-98 (1992).

33. Pan, W. et al. Resistance scaling for composite fermions in the presence of a density gradient. Preprint at http://arxiv.org/abs/cond-mat/0601627 (2006).

\section{Acknowledgements}

We thank D. Son and F. D. M. Haldane for alerting us of their theories and for invaluable discussions. We also thank J. Jain, N. P. Ong, R. Bhatt, M. Shayegan, D. Feldman, M. Levin, A. Stern, C. Kane, A. Viswanath, P. Zucker, S. Simon and M. Zudov for helpfu discussions. This work was supported by the US Department of Energy, Office of Science, Basic Energy Sciences, Materials Sciences and Engineering Division. Sandia National Laboratories is a multi-programme laboratory managed and operated by Sandia
Corporation, a wholly owned subsidiary of Lockheed Martin Corporation, for the US Department of Energy's National Nuclear Security Administration under contract DE-AC04-94AL85000. The work at University of Chicago was supported in part by the Templeton Foundation and NSF MRSEC Program through the University of Chicago

Materials Center. Sample growth at Princeton was funded by the Gordon and Betty

Moore Foundation through the EPiQS initiative GBMF4420, and by the National Science Foundation MRSEC Grant DMR-1420541.

\section{Author contributions}

W.P. and W.K. conceived the project; K.W.B., K.W.W. and L.N.P. grew the wafers for the experiment; W.P. did the measurements; W.P., W.K. and D.C.T. analysed the results and wrote the manuscript.

\section{Additional information}

Supplementary information is available in the online version of the paper. Reprints and permissions information is available online at www.nature.com/reprints. Publisher's note: Springer Nature remains neutral with regard to jurisdictional claims in published maps and institutional affiliations. Correspondence and requests for materials should be addressed to W.P. or W.K.

\section{Competing financial interests}

The authors declare no competing financial interests. 


\section{Methods}

Heterojunction insulated-gated field-effect transistors (HIGFETs) ${ }^{25}$ were exploited for measurements of the $\mathrm{SdH}$ oscillations and composite fermion conductivity. The growth structure of a typical HIGFET and the density dependence of mobility can be found in ref. 25 . A low-frequency $(\sim 11 \mathrm{~Hz})$ lock-in (Princeton Applied

Research 124A) technique was used to collect $R_{x x}$ and $R_{x y}$ as a function of electron density by sweeping the gate voltage at a fixed magnetic $(B)$ field. $\rho_{x x}$ is obtained from the measured $R_{x x}$ by taking into account the geometric ratio and $\rho_{x y}=R_{x y}$ in two dimensions.

Some of the unique, relevant aspects of HIGFETs are worth pointing out. In a HIGFET device, a heavily doped GaAs top layer serves as the top gate, which is a significant improvement over commonly used $\mathrm{Ti} / \mathrm{Au}$ or $\mathrm{Cr} / \mathrm{Au}$ gates for realizing a uniform $2 \mathrm{D}$ electron system. The differential thermal expansion between $\mathrm{Ti} / \mathrm{Au}$ (or $\mathrm{Cr} / \mathrm{Au}$ ) and $\mathrm{GaAs}$ is known to induce a severe electron density $\left(n_{\mathrm{e}}\right)$ inhomogeneity when the specimen is cooled from room temperature down to cryogenic temperatures. In addition, the insulating AlGaAs buffer layer in HIGFET devices is grown by molecular beam epitaxy (MBE), and considerably more uniform than the dielectric layers commonly used in field-effect transistors. The density of charge traps is reduced to the lowest level possible, and the linear relationship of $n_{\mathrm{e}}$ versus $V_{\mathrm{g}}$ can hold down to very low densities.

The spatial uniformity of electron density can be attested by the observation of a perfectly linear relationship in Supplementary Fig. 2d. Achieving a very low charge trap density in our HIGFET was confirmed by observing a perfect overlap between the two traces of $V_{\mathrm{g}}$ sweeping up and down. Finally, in all the HIGFET devices we studied, an electron mobility of $\mu \sim 1 \times 10^{6} \mathrm{~cm}^{2} \mathrm{~V}^{-1} \mathrm{~s}^{-1}$ was achieved at $n_{\mathrm{e}} \sim 2 \times 10^{10} \mathrm{~cm}^{-2}$, as shown in Supplementary Fig. 3. Such a high sample quality effectively eliminates disorder effects.

Data availability. The data that support the plots within this paper and other findings of this study are available from the corresponding authors upon reasonable request. 